\section{Severe isoniazid related sideroblastic anemia}

\author{
Rein Jan Piso, ${ }^{1}$ Kveti Kriz, ${ }^{2}$ \\ Marie-Claire Desax ${ }^{2}$
}

'Department of Medicine, Infectiology, Kantonsspital, Olten, Switzerland

2Department of Medicine, Haematology,

Kantonsspital, Olten, Switzerland

\begin{abstract}
Isoniazid induced sideroblastic anemia is a rare event. We report case of a 45 year old Caucasian women with development of severe anaemia 4 month after introduction of Isoniazid as part of Tuberculosis treatment. While haemoglobin fell to $47 \mathrm{~g} / \mathrm{L}$ and erythrocyte count to $1.5 \mathrm{G} / \mathrm{L}$, reticulocytes were very low (reticulocyte production index of 0.48 ), but bone marrow aspirate showed an accelerated erythropoiesis with ringsideroblasts. Anaemia rapidly resolved after cessation of Isoniazid. We postulate an Isoniazid induced inhibition of the $\delta$-Amino-levulinat-synthase resulting in marked depletion of heam synthesis.
\end{abstract}

\section{Case Report}

A 45-year old Caucasian women was diagnosed with tuberculous spondylitis and paraspinal abscess. M.tuberculosis could be isolated from intraoperativ biopsy; resistance testing revealed a completely sensitive strain. On July 2, treatment was started with rifampicin (RIF) $600 \mathrm{mg} /$ day, Isoniazid (INH) $300 \mathrm{mg} /$ day, Pyrazinamid (PZA) $1500 \mathrm{mg} /$ day and Ethambutol (ETB) $1200 \mathrm{mg} /$ day. After results of the resistance test where available, Ethambutol was stopped. Liver enzymes where elevated at one occasion but fell into normal range spontaneously. After two month of combination therapy RIF/INH/PZA, PZA was stopped according to international guidelines. Four month after begin of treatment, liver enzymes rose again, and INH was replaced by moxifloxacin. Liver enzymes fell to normal level, but the disturbed liver enzymes where later interpreted as alcohol induced. December 12 , progression of the paraspinal abscess was diagnosed, and the treatment was stopped for allowing new biopsies. On January 5, 2010, the patient was re-operated but mycobacteria couldn't be cultured in this second biopsy. Antimycobacterial treatment was reinstalled January 7. Because of possible non-compliance, we started again with a four drug combination for one month, followed by
INH/RIF/PZA. The patient then did not come to the visits until April 12. At that time the patient complaint about shortness of breath NYHA 2. Haemoglobin level had fallen to 47 gram/L with a mean cell volume of $96 \mathrm{fl}$ and a mean cell haemoglobin concentration of 334 $\mathrm{g} / \mathrm{L}$. PZA was stopped, but the other treatment was not changed. Two packs of red blood cells where transfused the day after. One week later, haemoglobin level had risen only to 51 gram/L. Bone marrow aspiration and biopsy was performed on April 20 (under Isoniazid-treatment). Isoniazid was substituted by moxifloxacin, rifampicin was not changed. Again two packs of red cell where transfused. Haemoglobin level rose to 89 gram/L, and reticulocyte production index rose from 0.48 to 2.9 one week after cessation of Isoniazid. No further transfusions where needed, and one week later, haemoglobin level was at 100 gram/L (Figure 1). Folic acid, Cobalamine and Ferritin values where in normal range, Parvovirus B19 PCR was negative. Bone marrow was not typical for PRCA, as it showed an enhanced, dysplastic erythropoiesis with $30 \%$ ringsideroblasts (Figures 2 and 3). No rearrangement of the MLL gene could be observed by fluorescent in situ hybridisation (FISH). Erythrocyte count in peripheral blood was $1.5 \mathrm{G} / \mathrm{L}$. Erythropoietin level was high, 1171 IU/L (normal range 12-23) and erythropoietin antibodies negative. Under treatment with rifampicin/moxifloxacin, haemoglobin levels returned to normal level and remained stable.

\section{Discussion}

Two major forms of anemia are decribed as side effect of treatment with Isoniazid. Pure red cell aplasia (PRCA), a syndrome characterized by severe normochromic normocytic anaemia, reticulocytopenia, and isolated erythroblastopenia in the bone marrow ${ }^{1}$ and sideroblastic anemia (SA), characterized by deficient haem synthesis and an increase of ringsideroblasts in bone marrow. Both are rare conditions. As the bone marrow did show an increased erythropoiesis with ringsideroblasts, we postulate a sideroblastic anemia. This disorder is generally divided in diseases of the haem synthesis pathway or of other mitochondrial pathways. ${ }^{2}$ Drug induced SA is due to a disorder in haem synthesis. ${ }^{2}$ Of tuberculostatic drugs, Isoniazid, Rifampin and Pyrazinamid are described as cause of SA. ${ }^{3-5}$ It is not clear if rifampin really induces $\mathrm{SA}$ alone. The reports show an SA in combination with Isoniazid, and Rifampin is frequently prescribed in prosthetic joint infections without supplementation of pyridoxine. If there would be a risk of development of SA by Rifampin alone, it should be seen more often in these situations. The fre-
Correspondence: Rein Jan Piso, Department of Medicine, Kantonsspital, 4600 Olten, Switzerland. E-mail: jpiso_ol@spital.ktso.ch

Key words: sideroblastic anaemia, isoniazid, tuberculosis.

Received for publication: 30 September 2010.

Revision received: 20 December 2010.

Accepted for publication: 14 January 2011.

This work is licensed under a Creative Commons Attribution 3.0 License (by-nc 3.0).

(C) Copyright R.J. Piso et al., 2011

Licensee PAGEPress, Italy

Hematology Reports 2011; 3:e2

doi:10.4081/hr.2011.e2

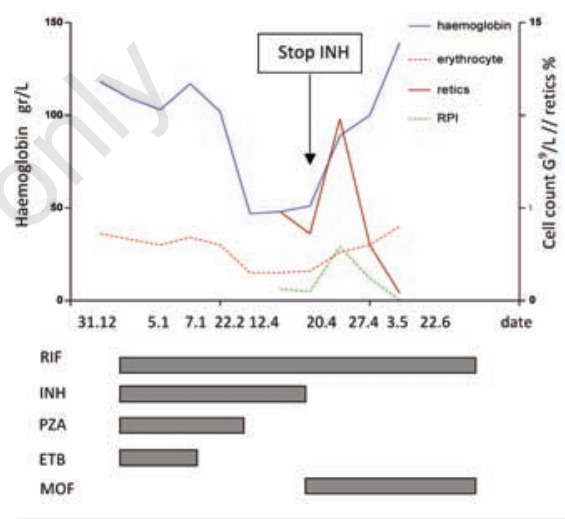

Figure 1. Blood values and treatment duration. RIF, rifampin; INH, isoniazid; PZA, pyrazinamide; ETB, ethambutol; MOF, moxifloxacin; RPI, reticulocyte production index.

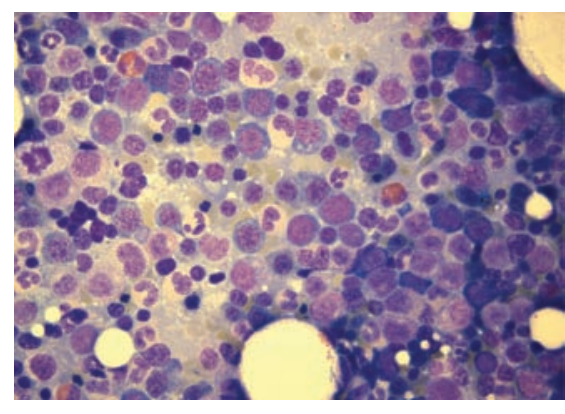

Figure 2. Hypercellular bone marrow aspirate with dysplastic erythropoiesis.

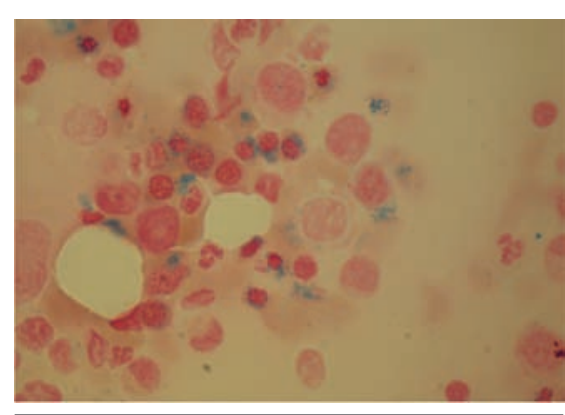

Figure 3. Ringsideroblasts. 
quency of Isoniazid induced SA is not clear, and only 8 cases are described in literature,, $35-10$ probably due to the generally prescribed comedication with pyridoxine. Some additional cases described as pyridoxine responsive anemia due to tuberculosis treatment may in fact also have been SA. ${ }^{11-13}$ The mechanism of Isoniazid induced SA is an inhibition of the $\delta$ aminolevulinate synthase-2 $2^{14}$ resulting in a depletion of haem synthesis. ${ }^{15}$ Pyridoxine acts as a co-factor in synthesis of $\delta$-aminolevulinate, and is inhibited by Isoniazid ${ }^{2}$ Substitution of pyridoxine is recommended during treatment with Isoniazid, and the patient was also treated according to these guidelines. ${ }^{16}$ While pyridoxine unresponsiveness has been described in hereditary SA, the situation in Isoniazid induced SA is not clear. In most cases, ${ }^{3,5,7,9}$ no pyridoxine was given during treatment with Isoniazid, and both supplementation and withdrawal of Isoniazid was performed after diagnosis of SA. We rather postulate a malcompliance in pyridoxine supplementation than a non responsiveness in our patient. Haden et al could demonstrate a steep rise in haemoglobin level with pyridoxine supplementation while the complete tuberculous treatment including Isoniazid was not changed. ${ }^{9}$ Although Isoniazid induced SA is a rare condition with known pathogenesis, clinicians should be aware of the potential side effect of the old drug regularly used for treatment of active and latent tuberculosis.

\section{References}

1. Krantz SB, Zaentz SD. Pure red cell aplasia. in Gordon A.S., Silber, R., LoBue, J. (eds): The year in Hematology 1977:15390.

2. Matthes T. Sideroblastic anemias. Ther Umsch 2006;63:25-33.

3. Sharp RA, Lowe JG, Johnston RN. Antituberculous drugs and sideroblastic anaemia. Br J Clin Pract 1990;44:706-7.

4. MacCurdy RK, Simon ER. Thrombo cytopenia and sideroblastic anemia with pyrazinoic acid amide (pyrazinamide) therapy. Chest 1970;57:378-81.

5. Vives JF, Rouy JM, Wagner A, Vallat G. Anemia with hypersideroblastosis during anti-tuberculosis therapy. cure with vitamin therapy]. Nouv Rev Fr Hematol 1978; 20:99-110.

6. Liu PI, Lim JY. Sideroblastic anemia. JAMA 1987;257:2814.

7. Yoshimoto S, Takeuchi M, Tada A. Secondary sideroblastic anemia caused by long term administration of anti-tuberculous agents including isoniazid]. Rinsho Ketsueki 1992;33:986-90.

8. Chrobak L. Sideroblastic anemia after treatment with isoniazid (nidrazid)]. Vnitr Lek 1974;20:563-6.

9. Haden HT. Pyridoxine-responsive sideroblastic anemia due to antituberculous drugs. Arch Intern Med 1967;120:602-6.

10. Tomkin GH. Isoniazid as a cause of neuropathy and sideroblastic anaemia. Practitioner 1973;211:773-7.

11. McCurdy PR, Donohoe RF. Pyridoxineresponsive anemia conditioned by isonicotinic acid hydrazide. Blood 1966;27:352-62.

12. Horrigan DL, Harris JW. Pyridoxineresponsive anemia: analysis of 62 cases. Adv Intern Med 1964;12:103-74.

13. Harris JW, Whittington RM, Weisman R Jr, Horrigan DL. Pyridoxine responsive anemia in the human adult. Proc Soc Exp Biol Med 1956;91:427-32.

14. Pasanen AV. The activities of delta-aminolaevulinic acid synthase and haem synthase in experimental sideroblastic anaemia. effect of mitochondrial iron excess on the enzyme activity in peripheral red blood cells. Scand J Clin Lab Invest 1981;41:159-65.

15. Chalevelakis G, Yalouris AG, Lyberatos C, et al. Effect of isoniazid, a haem inhibitor, on globin chain synthesis in reticulocytes from non-thalassaemic and beta thalassaemic subjects. J Clin Pathol 1989;42:97781.

16. American Thoracic Society, CDC, Infectious Diseases Society of America. Treatment of tuberculosis. MMWR Recomm Rep 2003;52:1-77. 